despite receiving Intravenous antibiotics for presumed partially treated meningitis. He also received IVIG for the suspicion of atypical Kawasaki. Computerised tomography of the head and abdomen and bone marrow studies were also inconclusive. The mother subsequently revealed that a previous child had died during infancy with prolonged fever and no specific diagnosis. This alerted the treating physicians to the possibility of immunodeficiency. Our patient had received BCG vaccine at birth without any complications, and three gastric aspirate samples were negative for tuberculosis. A broncho-alveolar lavage was performed, despite the absence of any respiratory symptoms, to exclude any unusual organisms causing prolonged fever. The broncho-alveolar lavage revealed mycobacterium tuberculosis which was resistant to Isoniazid and the patient was started on appropriate antiTuberculous medications. The total immunoglobulin counts and Flow cytometry were suggestive of SCID.

Conclusion Pulmonary Tuberculosis should be considered as a differential diagnosis of FUO, especially in infants with suspected or proven SCID. Attaining a good family history is crucial in early diagnosis of SCID and hence possibly in saving lives.

\section{P0-0009 INCREASED PNEUMOCOCCUS RESISTANCE TO ANTIBIOTICS AND DIFFICULTIES REGARDING THERAPEUTIC OPTIONS}

1,2S lurian. 'Clinical Laboratory, Pediatric Hospital, Sibiu, Romania; ${ }^{2}$ Pediatric Clinic, Lucian Blaga University, Sibiu, Romania

\subsection{6/archdischild-2014-307384.689}

Background During childhood pneumococccus (S.pneumoniae) is identified not only in nasopharynx (carriers), but also as etiological agent in conjunctivitis, respiratory airways infections, meningitis etc. Choosing the optimal therapy is difficult due to: prolonged time necessary to antibiotic sensitivity test (AST) achievement, difficulty to perform it and pneumococcus strains growing resistance. In Romania vaccination against S.pneumoniae is not offered in routine immunisation program.

Aims 1. To analyse resistance spectrum for S.pneumoniae strains; 2. Establishing empirical therapeutic decisions according to pneumococcus resistance profile in our county.

Methods During October 2011-January 2014, there were performed AST for S.pneumoniae using Vitek AST-P576 cards. Samples origin10 nasal secretions, 21 conjunctival secretions, 12 ear samples, 4 hemocultures, 1 cerebrospinal fluid sample, 1 urine culture. Correlated with minimal inhibitory concentration to penicillin, strains were divided into: sensitive, intermediate, resistant (penicillin resistant pneumococcus-PRP). Authors took also into consideration for AST: Amoxicillin (AMX), Cefotaxime (CTX), Ceftriaxone (CRO), Eritromicine (E), Levofloxacin (L), Vancomycin (VA), Trimethoprim-Sulfamethoxazole (SXT).

Results Among 51 validated AST, authors noticed: 58.8\% Penicillin resistance (30 PRP strains); 43.7\% AMX resistance; $38 \mathrm{E}$ resistant strains $(82.6 \%) ; 74.5 \%$ SXT resistance; for CRO resistance reached $33.3 \% ; 21.5 \%$ CTX resistance. All strains were sensitive for L, VA.

Conclusions 1. Authors observed increased resistance to penicillin, E and STX in our county; 2. Therapeutic options are limited in S.pneumoniae infection; 3. According to resistance pattern, authors restrict use of macrolides and STX in otitis media (most frequent etiological agent is S.pneumoniae); 4. In severe infections, therapeutic choices are CTX, CRO, VA; 5. Implementation of educational measures is important in order to avoid antibiotic overuse.

\section{PO-0010 THE PHYSICIANS APPROACH TO} COMMUNITY-ACQUIRED PNEUMONIA IN CHILDHOOD

'A Yazar, ${ }^{1} \mathrm{H}$ Aydogan, 'S Guven, ${ }^{1} \mathrm{H}$ Saner, ${ }^{1} \mathrm{I}$ Islek, ${ }^{2} \mathrm{E}$ Pala. ${ }^{1}$ Pediatrics, Umraniye Training and Research Hospital, Istanbul, Turkey; ${ }^{2}$ Family Medicine, Umraniye Training and Research Hospital, Istanbul, Turkey

\subsection{6/archdischild-2014-307384.690}

Background and aims Diagnosis and treatment guidelines for community-acquired pneumonia (CAP) are prepared to avoid differences for the diagnosis and management of CAP between physicians. We investigated the approaches of physicians to diagnosis, laboratory findings, treatment and the compatibility with guidelines for CAP.

Method A total of 322 doctors were interviewed face to face and a 12 item-questionary incluiding diagnosis, investigation, treatment criteria and drug choices was filled by the physician.

Results Contrarily to the guidelines $6.9 \%$ of physicians preferred auscultation and chest radiographygraphy (CXR) and did not use the symptoms for diagnosis. Only $11.8 \%$ preferred symptoms and diagnostic investigations. $58.8 \%$ did not use CXR, CBC and CRP, 24.5\% did not use CXR, CBC, CRP and ERS and $4.9 \%$ of them did not prefer any investigations. Also routine CXR to confirm CAP in outpatient setting children is not recommended by guidelines physicians preferred CXR at high percentage. Physicians did not preferred Amoksisilin for mild CAP between 3 months - 2 years old children, 55\% preferred parenteral treatment with ampisilin/sulbactam. Parenteral Seftriakson was preferred for hospitalised patients ( $>5$ years) with severe pneumonia. $49.7 \%$ of physicians preferred ten day duration of therapy, $17.1 \%$ stopped treatment after disappearance of symptoms and auscultation findings, $13.7 \%$ completed the treatment after improvement of CXR.

Conclusion Physicians applied different approaches to the diagnosis and treatment of CAP in infants and children. According to our findings, we suggested that physicians should be educated about approaches for treatment of CAP according to guidelines.

\section{PO-0011 LENGTH OF STAY IN INFANTS AND CHILDREN HOSPITALISED WITH ACUTE BRONCHIOLITIS: RSV VERSUS NON-RSV}

M Khalifa, A Al Hamadi, M Hendaus, E Muneer. General Pediatrics, Hamad Medical Corporation, Doha, Qatar

\subsection{6/archdischild-2014-307384.691}

Background Respiratory Syncythial Virus (RSV) is a common lower respiratory tract viral infection. RSV and a wide variety of other respiratory viruses are common triggers for bronchiolitis.

Aim To determine the length of stay in infants with acute RSV bronchiolitis vs. Non-RSV.

Materials and methods A retrospective study was conducted at Hamad Medical Corporation (HMC). Infants and children ages 0 to 18 months hospitalised with acute bronchiolitis from October 2010 to March 2013 were included. The data collected: age at diagnosis, sex, direct fluorescent antibody (DFA) and length of stay.

Results The study included 838 infants, mean age 3.6( \pm 3.5$)$ months, and boys constituted 60\%. DFA was conducted on 770 infant, where 352 Were RSV positive (45.7\%), 142 were RSV negative $(18.4 \%)$ and other non-RSV viruses (Adenoviurs, RhinoVirus, InfluenzaVirus, Parainflenzavirus, and Bocavirus)276 (35.8\%). The mean length of stay for RSV-positive was 8.03 days, 95\% C. I. (7.26-8.79), and 6.94 days, 95\% C. I. (5.89- 